\title{
Challenges of Constructing a Multiple-Perspective Domain Analysis of Neurodiversity
}

\author{
Annuska Zolyomi \\ University of Washington \\ Seattle, Washington \\ annuska@uw.edu
}

\begin{abstract}
When constructing a domain analysis of a phenomenon, researchers will likely encounter complexity in the method and resulting outcome, such as a taxonomy or thesaurus. This paper reflects upon a recent endeavor to conduct a domain analysis of neurodiversity with a focus on Autism Spectrum Disorder. Through the construction of this multi-faceted taxonomy, the researchers faced challenges regarding framing the perspectives represented by the taxonomy and addressing gaps and changes in the taxonomy. These challenges raise methodological, ethical, and technical issues. Ultimately, the researchers' biases are inherent in their editorial decisions and are influential, yet often hidden, factors in the domain analysis.
\end{abstract}

\section{Keywords}

Domain analysis, neurodiversity, autism.

\section{INTRODUCTION}

This conceptual paper examines the research process behind "Autism Prism: A Domain Analysis Examining Neurodiversity" (Zolyomi and Tennis 2017). That prior work aimed to capture a snap-shot of the phenomenon of neurodiversity, with an emphasis on Autism Spectrum Disorder (ASD). The resulting taxonomy reflected two primary models of neurodiversity: the medical model and the social model. Through the construction of this multi-faceted taxonomy, the researchers faced challenges regarding framing the perspectives represented by the taxonomy and addressing perceived gaps in the taxonomy. These challenges are an opportunity to refine domain analysis methods and to clarify ethical stances. They also raise open issues about the technical challenges of maintaining a dynamic taxonomy whose terms and sources will change over time. This paper provides examples from the Neurodiversity domain analysis to illustrate these challenges. Ultimately, the researcher's bias is inherent in their method and analysis. This paper proposes that a

Advances in Classification Research, 2017, October 27, 2017, Washington, DC, USA. statement of the researcher's epistemological and ontological stance would benefit both the researcher and reader.

\section{FRAMING PERSPECTIVES}

The neurodiversity domain analysis aimed to detangle the myriad of voices, often with conflicting viewpoints and information, regarding autism in the health, social justice, education, academic, and public discourse. One challenge of constructing a taxonomy is selecting which overarching perspectives will be represented in the taxonomy. In the case of disability-related taxonomies, Disability Studies can provide useful scholarship. Disability Studies scholars define two primary models, the medial and social models, to emphasize functional-limitations versus societal-limitations, respectively (Anastasiou and Kauffman 2013; Oliver 1990). Although it was useful to scope our analysis to the two primary models, it was limiting in three key ways. First, it simplified the scholarly work of Disability Studies, which is more nuanced. Some scholars reserve the use of medical model to characterize the over medicalization of a disability, which results in focusing on curing a disability as an illness. They may use the alternative term for medical model, "individual model," when articulating a viewpoint of disability being located within the individual. Second, although the social model is viewed as empowering autistic individuals and their families, there is, naturally, not just one, unifying opinion held by everyone in the autism community. The third limiting result was that people and organizations do not necessarily fit into just one model. For instance, a disability pediatric doctor may also be a parent of an autistic child. An organization may first be focused on advocating for a cure and later change course to advocating for educational services. These limitations resulted in a loss of fidelity and potential insights in the resulting taxonomy.

After choosing the primary models, the researchers then needed to select the organizations, institutions, scholarly work, and individuals that best represent and bring life to those models. For curating sources, researchers need to create metrics for credible and influential sources. In our case, we selected the U.S. standard for medical diagnosis, the Diagnostic and Statistical Manual of Mental Disorders (DSM), 5th edition (American Psychiatric Association 2013). As a secondary source, we used the World Health Organization's International Statistical Classification of Diseases and Related Health Problems (ICD-10) (World Health Organization 2010). However, for a non-U.S. focused 
analysis, it would have been difficult to choose which source should be primary and secondary. Many of our sources were online resources, including an alternative dictionary, the "Double-Tongued Dictionary Index" (2016). We established the criteria that any online source needed to be crossreferenced by at least one other source and that the author needed to have self-identified as being autistic. This criteria and process should be examined further, especially as more online content is created in many different forms (e.g., blogs, YouTube videos, social media). In addition, this process privileges people and groups who have access and abilities to create online content. Some members of the autism community are non-verbal, and some individuals also have cognitive impairments. To capture a holistic representation of the population, our research method should account for differences in communication styles and abilities.

\section{PERCEIVED GAPS AND CHANGES IN TAXONOMY}

After collecting and identifying the relationships between the taxonomy terms, the researchers identified gaps in the taxonomy. These gaps were evidenced by missing terms and by concepts that were discussed but not explicitly named. For instance, individuals talked about their pathway to an official diagnosis. Some people were self-diagnosed or communitydiagnosed. Yet there was a phase just prior during which the individual was exploring their neurodiversity. We determined that this phase warranted naming and proposed the term "neuro-curious." This action raises the question of the role of the researcher in constructing a taxonomy and generating new terms. By placing neuro-curious in the taxonomy, we gave it an equal weight as community-named terms, when perhaps the term should have been differentiated as researcher-defined.

Another issue related to the dynamic nature of taxonomies is that terms and their relationships change over time. Terms fade as they become out of fashion, become discriminatory, or are officially removed, such as the case of the DSM-IV Asperger's diagnosis. New terms arise as people's identities evolve and they create or embrace different nomenclature. The terms "neurodiverse" and "autistic" are used by young adults on the autism spectrum as a statement of pride and autonomy. As terms emerge as the community evolves and scientific knowledge deepens, it is unclear how a taxonomy should record its historic and emergent terms.

\section{RESEARCHER STANCE}

Throughout the process of conducting a domain analysis, the researcher is making decisions about framing the multiple perspectives, curating sources, analyzing gaps, and forefronting key tensions between perspectives. The researcher's epistemological and ontological stance will influence these decisions, and therefore, the resulting taxonomy. In conducting analysis of cultures and communities, a researcher may be a member of the community, an ally of the community or an outsider to the community. Similar to ethnographic research, the researcher's relationship to the community can open or restrict access to the true nature of the community, including terms and opinions kept within the community (Guimaraes et al. 2017). Both the researcher and reader would benefit from an expression of the researcher's stance and evaluation of potential bias.

\section{CONCLUSION}

Constructing a taxonomy and analysis of the neurodiversity domain was an insightful and productive research process. This dynamic, multi-perspective nature of neurodiversity has parallels with domains related to disability, health, cultural, and intersectional identities (Fox 2017). The case study of a neurodiversity domain analysis illustrated methodological, ethical, and technical challenges of constructing and maintaining a multi-perspective taxonomy. By sharing these challenges, we hope to contribute to ongoing dialog in the Classification community to better understand these challenges and formulate promising solutions.

\section{REFERENCES}

American Psychiatric Association. (2013). Diagnostic and Statistical Manual of Mental Disorders, Fifth Edition. Arlington, VA: Author.

Anastasiou, D., and J. M. Kauffman. (2013). The Social Model of Disability: Dichotomy between Impairment and Disability. Journal of Medicine and Philosophy 38(4): 441-59. doi:10.1093/jmp/jht026

Double-Tongued Dictionary Index | A Way with Words. (2016). Retrieved December 24, 2016. https://www.waywordradio.org/dictionary-listing/.

Fox, M. J. (2017). Sorting the Unsortable: Visualizing Intersectionality in Knowledge Organization. North American Symposium on Knowledge Organization, University of Illinois at Champaign-Urbana. June 15-16, 2017.

Insel, T., Cuthbert, B., Garvey, M., Heinssen, R., Pine, D. S., Quinn, K., ... Wang, P. (2010). Research domain criteria (RDoC): toward a new classification framework for research on mental disorders. American Journal of Psychiatric 167(7), 748-751.

Guimaraes, J.A.C, Pinho, F.A., Martinez-Avila, D., Campbell, G., and Nascimento, F.A. (2017). Knowledge Organization and the Power to Name: LGBTQ Terminology and the Polyhedron of Empowerment. North American Symposium on Knowledge Organization, University of Illinois at Champaign-Urbana. June 15-16, 2017.

Oliver, Mike. (1990). The Individual and Social Models of Disability. Retrieved July 23. http://pf7d7vi404s1dxh27mla5569.wpengine.netdnacdn.com/files/library/Oliverin-soc-dis.pdf 
World Health Organization. (2010). International Statistical Classification of Diseases and Related Health Problems (ICD-10), 10th Revision. Retrieved from http://apps.who.int/classifications/icd10/browse/2010/en\# /F80-F89.
Zolyomi, A. \& Tennis, J. (2017). The Autism Prism: A Domain Analysis of Neurodiversity. Proceedings of the North American Symposium on Knowledge Organization 6, 139-172. 